\title{
Direct Maternal Death
}

National Cancer Institute

\section{Source}

National Cancer Institute. Direct Maternal Death. NCI Thesaurus. Code C111858.

Death resulting from conditions or complications which are unique to pregnancy and occur during the antepartum, intrapartum, or postpartum period. 